\title{
Structural Change of Cooper Pairs and Momentum-dependent Gap in Color Superconductivity
}

\author{
Hiroaki Abuki* and Tetsuo Hatsuda ${ }^{\dagger}$ \\ Department of Physics, University of Tokyo, Tokyo 113-0033, Japan \\ Kazunori Itakura ${ }^{\ddagger}$ \\ RIKEN BNL Research Center, Brookhaven National Laboratory, Upton, NY 11973, USA
}

(October 30, 2018)

\begin{abstract}
The two-flavor color superconductivity is studied over a wide range of baryon density with a single model. We pay a special attention to the spatial-momentum dependence of the gap and to the spatial-structure of Cooper pairs. At extremely high baryon density $\left(\sim \mathcal{O}\left(10^{10} \rho_{0}\right)\right.$ with $\rho_{0}$ being the normal nuclear matter density), our model becomes equivalent to the usual perturbative QCD treatment and the gap is shown to have a sharp peak near the Fermi surface due to the weak-coupling nature of QCD. On the other hand, the gap is a smooth function of the momentum at lower densities $\left(\sim \mathcal{O}\left(10 \rho_{0}\right)\right)$ due to strong color magnetic and electric interactions. To study the structural change of Cooper pairs from high density to lower density, quark correlation in the color superconductor is studied both in the momentum space and in the coordinate space. The size of the Cooper pair is shown to become comparable to the averaged inter-quark distance at low densities. Also, effects of the momentum-dependent running coupling and the antiquark pairing, which are both small at high density, are shown to be non-negligible at low densities. These features are highly contrasted to the standard BCS superconductivity in metals.
\end{abstract}

\section{INTRODUCTION}

Because of the asymptotic freedom and the Debye screening in QCD, deconfined quark matter is expected to be realized for baryon densities much larger than the normal nuclear matter density [1]. Furthermore, any attractive quark-quark interaction in the cold quark matter causes an instability of the Fermi surface by the formation of Cooper pairs and leads to the color superconducting phase [2] 6 .

Current understanding on the color superconductivity has been based on two different theoretical approaches. One is an analysis by the one-loop Schwinger-Dyson equation with perturbative one gluon exchange, which is valid at asymptotically high densities [7] 9. The dominant contribution to the superconducting gap at very high density comes from the collinear scattering through the long range magnetic gluon exchange [10]. In such a weak-coupling regime, formation of Cooper pairs takes place only in a small region near the Fermi surface. The other approach to the color superconductivity is the mean-field approximation with QCD inspired 4-Fermi model which is introduced to study lower density regions [4,5, 11, 12]. In this approach, magnitude of the gap becomes as large as $100 \mathrm{MeV}$ and is almost constant in the vicinity of Fermi surface as a function of momentum.

The main purpose of this paper is to calculate the superconducting gap over a wide range of baryon density with a single model and to make a bridge between high and low density regimes. In particular, we make an extensive analysis on the structural change in spatial-momentum dependence of Cooper pair from high to low densities. At relatively low baryon densities, the gluonic attraction becomes effective for all quarks inside the Fermi sea and sizable Cooper pairing takes place for wide range of momentum away from the Fermi surface. This is in contrast to the phonon interaction with an intrinsic Debye cutoff in the BCS-type superconductivity [13]. The spatial-momentum dependence of the gap, diffuseness of the Fermi surface, quark-quark correlations in the superconductor and the spatial size of Cooper pairs are the characteristic quantities which reflect the departure from the weak-coupling picture. So far, only a few investigations have been made along this line [3, 14, 15].

Throughout the paper, we limit our discussions to 2-flavor color superconductivity partly because our primary interests are in low densities and partly because the analysis is simpler than in the three-flavor case [16]. To investigate how the weak-coupling picture is modified at low densities in a qualitative manner, we adopt a model called the

\footnotetext{
*abuki@nt.phys.s.u-tokyo.ac.jp

${ }^{\dagger}$ hatsuda@phys.s.u-tokyo.ac.jp

$\ddagger_{\text {itakura@bnl.gov }}$
} 
improved ladder approach in which the one-loop Schwinger-Dyson equation with infrared safe running coupling is used. This model is known to reproduce the physics of the QCD vacuum and the $q \bar{q}$ meson properties reasonably well 17 19. For recent applications of similar kind of models to QCD at finite temperature and density, see the review [21. As we will show later, the model can naturally reproduce the correct asymptotic behavior of the superconducting gap, in the high density limit. Therefore, it is a suitable model for our purpose to study the color superconductivity for a wide range of density. To make our analysis as simple as possible, we use a gluon propagator with a minimal static and dynamical corrections (Debye screening and Landau damping). The similar form of the gluon propagator is also adopted in Ref. 222.

This paper is organized as follows. In Sect. II, we define our model and derive relevant gap equations. We first consider the weak-coupling region at high density and discuss the general properties of the gap as a function of the spatial-momentum. In Sect. III, we solve the momentum-dependent gap equation numerically for a wide range of density. A large structural change of the momentum-dependent gap will be shown. The quark and antiquark occupation numbers, the correlation of quarks in the color superconductor, and the coherence length are also investigated. Summary and concluding remarks are given in the last section. In Appendix A, the gauge dependence of the gap equation is discussed. In Appendix B, we derive the asymptotic behavior of the spatial quark-quark correlation in the weak coupling.

\section{GAP EQUATION WITH SPATIAL MOMENTUM DEPENDENCE}

In this section, we define our model and derive relevant gap equations in two flavor QCD with the one-gluon exchange interaction. We start with the standard Schwinger-Dyson equation for the quark self-energy with four momentum dependence [7]. Then it is reduced to a gap equation with spatial-momentum dependence. General properties of the solution of our gap equation in the weak-coupling limit are also examined. The gap with spatial-momentum dependence enables us to study the diffuseness of the Fermi surface and the the structure of Cooper pairs in the color superconductor.

Throughout this paper, we use the following notation for the four and three momenta : $k^{\rho}=\left(k^{0}, \boldsymbol{k}\right), k=|\boldsymbol{k}|, \hat{\boldsymbol{k}}=$ $\boldsymbol{k} / k$. Also, we limit ourselves to the system at zero temperature and work in the Minkowski space with the quark and gluon propagators obeying the Feynman boundary condition.

\section{A. The model}

Let us start with the definition of quark self-energy and the superconducting gap. Using the standard NambuGor'kov formalism with a two component Dirac spinor $\Psi=\left(\psi, \bar{\psi}^{t}\right)$, the quark self-energy $\Sigma\left(k_{\rho}\right)$ with the Minkowski 4 -momentum $k_{\rho}$ is written as

$$
\Sigma\left(k_{\rho}\right)=S_{0}^{-1}\left(k_{\rho}\right)-S^{-1}\left(k_{\rho}\right)=\left(\begin{array}{cc}
0 & \bar{\Delta}\left(k_{\rho}\right) \\
\Delta\left(k_{\rho}\right) & 0
\end{array}\right),
$$

where the superconducting gap $\Delta$ and $\bar{\Delta} \equiv \gamma_{0} \Delta^{\dagger} \gamma_{0}$ enter through the off-diagonal components of $\Sigma$. The diagonal components of $\Sigma$ is neglected throughout this paper [7. $S\left(k_{\rho}\right)$ and $S_{0}\left(k_{\rho}\right)$ are the full and free quark propagators, respectively. The free propagator is taken to be a form $S_{0}^{-1}\left(k_{\rho}\right)=\operatorname{diag}$. $\left(\not k+\not k,(\not k-\not k)^{T}\right)$. We ignore the mass of $u$ and $d$ quarks.

In the ladder approximation of the one gluon exchange, the Schwinger-Dyson equation for $\Sigma$ is written as

$$
\Sigma\left(k_{\rho}\right)=-i \int \frac{d^{4} q}{(2 \pi)^{4}} g^{2} \Gamma_{a}^{\mu} S\left(q_{\rho}\right) \Gamma_{b}^{\nu} D_{\mu \nu}^{a b}\left(q_{\rho}-k_{\rho}\right),
$$

where $D_{\mu \nu}^{a b}$ is the gluon propagator in medium and $\Gamma_{\mu}^{a}$ is the quark-gluon vertex.

To study the flavor anti-symmetric, color anti-symmetric and $J=0^{+}$channel (which is the most attractive channel within the one gluon exchange model), we assume the following structure of the gap function [7] 9.23],

$$
\Delta\left(k_{\rho}\right)=\left(\lambda_{2} \tau_{2} C \gamma_{5}\right)\left(\Delta_{+}\left(k_{\rho}\right) \Lambda_{+}(\hat{\boldsymbol{k}})+\Delta_{-}\left(k_{\rho}\right) \Lambda_{-}(\hat{\boldsymbol{k}})\right) .
$$

Here $\tau_{2}$ is the Pauli matrix acting on the flavor space, $\lambda_{2}$ is a color anti-symmetric Gell-Mann matrix, and $C$ is the charge conjugation. $\Lambda_{ \pm}(\hat{\boldsymbol{k}}) \equiv(1 \pm \hat{\boldsymbol{k}} \cdot \boldsymbol{\alpha}) / 2$ is the projector on positive $(+)$ and negative $(-)$ energy quarks. $\Delta_{ \pm}$will be identified as the gap in quark and antiquark channel, respectively. 
For the spinor and color structures of the vertex in Eq. (2.2), we use the bare vertex $\Gamma_{\mu}^{a}=\operatorname{diag} .\left(\gamma_{\mu} \lambda^{a} / 2,-\left(\gamma_{\mu} \lambda^{a} / 2\right)^{t}\right)$ [7]. For $g^{2}$ in Eq. (2.2), we use a momentum dependent coupling $g^{2}(q, k)$ which does not diverge at low momentum scale. In the "improved ladder approximation" $17, g^{2}(q, k)$ is taken to be

$$
g^{2}(q, k)=\frac{8 \pi^{2}}{\beta_{0}} \frac{1}{\ln \left(\left(p_{\max }^{2}+p_{c}^{2}\right) / \Lambda^{2}\right)}, \quad p_{\max }=\max (q, k),
$$

where $\beta_{0}=\left(11 N_{c}-2 N_{f}\right) / 3, p_{c}^{2}$ plays a role of a phenomenological infrared regulator, and $\Lambda$ dictates the logarithmic decreases of the vertex at high momentum. The quark propagator in the improved ladder approximation in the vacuum is known to have a high momentum behavior consistent with that expected from the renormalization group and the operator product expansion. For the numerical values of $\Lambda$ and $p_{c}^{2}$, we adopt $400 \mathrm{MeV}$ and $1.5 \Lambda^{2}$ respectively. They are determined to reproduce the low energy meson properties for $N_{f}=2$ by solving the Schwinger-Dyson equation for the quark propagator and the Bethe-Salpter equation for the $q \bar{q}$ bound state in the vacuum [18].

We use the following gluon propagator in medium in Eq. (2.2) in the Landau gauge,

$$
D_{\mu \nu}\left(k_{\rho}\right)=-\frac{P_{\mu \nu}^{\mathrm{T}}}{\boldsymbol{k}^{2}+i M^{2}\left|k_{0}\right| /|\boldsymbol{k}|}-\frac{P_{\mu \nu}^{\mathrm{L}}}{\boldsymbol{k}^{2}+m_{\mathrm{D}}^{2}},
$$

where $P_{\mu \nu}^{\mathrm{T}, \mathrm{L}}$ are the transverse and longitudinal projectors satisfying the relations, $P_{i j}^{\mathrm{T}}=\delta_{i j}-\hat{k}_{i} \hat{k}_{j}, P_{00}^{\mathrm{T}}=P_{0 i}^{\mathrm{T}}=0$, and $P_{\mu \nu}^{\mathrm{L}}=-g_{\mu \nu}+k_{\mu} k_{\nu} / k_{\rho}^{2}-P_{\mu \nu}^{\mathrm{T}}$. The longitudinal part of the propagator has static screening by the Debye mass $m_{\mathrm{D}}^{2}=\left(N_{f} / 2 \pi^{2}\right) g^{2} \mu^{2}$, while the transverse part has dynamical screening by the Landau damping $M^{2}=(\pi / 4) m_{\mathrm{D}}^{2}$ [20]. The above form of the propagator is a quasi-static approximation of the full gluon propagator in the sense that only the leading frequency dependence is considered. The same form is also adopted in Ref. [22].

Here we make a brief comment on the gauge-parameter $\xi$ dependence of the gap equation. Although physical quantities should be gauge invariant, special truncation scheme of the diagrams such as the ladder approximation introduces gauge-parameter dependence. It has been claimed that (i) $\Delta_{+}$is gauge invariant at extremely high density [7], and (ii) the gauge dependent contribution only begins to decrease at extraordinarily high densities $\mu>10^{8} \mathrm{MeV}$ and seems to converge to the result of the Landau gauge $(\xi=0)$ [24]. We will show in Appendix A that, if one appropriately treats the momentum dependence, the $\xi$-dependence survives in the gap equation for $\Delta_{+}$but gives a sub-leading contribution. Under this remark, we will consider only the Landau gauge $(\xi=0)$ throughout this paper.

The gap equation under the approximations shown above is obtained from the 2-1 element of the Schwinger-Dyson (matrix) equation (2.2). Then the final form of the gap equation for $\Delta_{ \pm}\left(k_{\rho}\right)=\Delta_{ \pm}\left(k_{0}, \boldsymbol{k}\right)$ reads

$$
\begin{aligned}
\Delta_{ \pm}\left(k_{\rho}\right)= & \frac{N_{c}+1}{2 N_{c}} i \int \frac{d^{4} q}{(2 \pi)^{4}} g^{2}(q, k)\left\{\frac{1}{2} \operatorname{tr}\left(\Lambda_{ \pm}(\hat{\boldsymbol{k}}) \gamma^{\mu} \Lambda_{-}(\hat{\boldsymbol{q}}) \gamma^{\nu}\right) \frac{\Delta_{+}\left(q_{\rho}\right)}{q_{0}^{2}-E_{+}^{2}(q)-\left|\Delta_{+}\left(q_{\rho}\right)\right|^{2}}\right. \\
& \left.+\frac{1}{2} \operatorname{tr}\left(\Lambda_{ \pm}(\hat{\boldsymbol{k}}) \gamma^{\mu} \Lambda_{+}(\hat{\boldsymbol{q}}) \gamma^{\nu}\right) \frac{\Delta_{-}\left(q_{\rho}\right)}{q_{0}^{2}-E_{-}^{2}(q)-\left|\Delta_{-}\left(q_{\rho}\right)\right|^{2}}\right\} D_{\mu \nu}\left(q_{\rho}-k_{\rho}\right),
\end{aligned}
$$

where $E_{ \pm}(q)(=q \mp \mu)$ is the reduced energy for free quarks (for + sign) and for antiquarks (for - sign).

\section{B. Analytic properties of the gap in the weak coupling}

\section{Gap equation in the weak-coupling limit}

Let us first study the analytic properties of the gap equation with spatial-momentum dependence. To obtain analytically tractable equations, we consider here the weak-coupling (high density) limit. This "weak-coupling approximation" implies the approximation where we take only the color-magnetic interaction and the real part of $\Delta_{+}$. Momentum dependence of $g^{2}(q, k)$ is neglected. Contributions from the antiquarks proportional to $\Delta_{-}$and the momentum-dependent factor originating from the projection operator in Eq. (2.6) are also neglected. We also ignore the imaginary part of the propagator because it is factor $g^{2}$ smaller than the real part. In Eq. (2.6), the frequency integral is performed by picking up quasi-particle poles in the quark propagator. After integrating over the angular variable, we obtain the following gap equation with the spatial-momentum dependence

$$
\Delta_{+}(k)=\frac{g^{2}}{144 \pi^{2}} \int_{0}^{\infty} d q \frac{\Delta_{+}(q)}{\sqrt{E_{+}^{2}(q)+\Delta_{+}^{2}(q)}}\left(\frac{q}{k} \ln \left[\frac{(q+k)^{6}+M^{4}\left(\epsilon_{k}^{+}-\epsilon_{q}^{+}\right)^{2}}{(q-k)^{6}+M^{4}\left(\epsilon_{q}^{+}-\epsilon_{k}^{+}\right)^{2}}\right]+\left(\epsilon_{q}^{+} \rightarrow-\epsilon_{q}^{+}\right)\right)
$$


where $E_{+}(q) \equiv q-\mu$ and we have defined $\Delta_{+}(q) \equiv \Delta_{+}\left(\epsilon_{q}^{+}, q\right)$ for notational simplicity. $\epsilon_{q}^{+}$is the quasi-particle energy as a solution of $\left(\epsilon_{q}^{+}\right)^{2}=E_{+}^{2}(q)+\Delta_{+}^{2}\left(\epsilon_{q}^{+}, q\right)$. This is the final form of an approximate gap equation in the weak-coupling limit. $\Delta_{+}(k)$ corresponds to the gap of the on-shell quasi-particles in the color superconductor since $k_{0}$ is taken to be $\epsilon_{k}^{+}$.

\section{Gap near the Fermi surface in weak coupling limit}

Let us consider the behavior of the gap near the Fermi surface. As we claimed in Introduction, the gap equation (2.7) reproduces the correct high density solution near the Fermi surface, which was previously derived in Refs. [7. 10] for frequency dependent gap, and in Ref. [] for momentum dependent gap. For this purpose, we pick up the most singular part of the integrand by taking $q=k=\mu$ in the bracket except for the term $\left(\epsilon_{q}^{+}-\epsilon_{k}^{+}\right)^{2}$ in the denominator. Then Eq. (2.7) reduces to

$$
\Delta_{+}(k \sim \mu)=\frac{g^{2}}{72 \pi^{2}} \int_{0}^{\infty} d q \frac{\Delta_{+}(q)}{\sqrt{E_{+}^{2}(q)+\left|\Delta_{+}(q)\right|^{2}}} \ln \frac{(b \mu)^{2}}{\left|\epsilon_{q}^{+2}-\epsilon_{k}^{+2}\right|},
$$

where $b=2(2 \mu / M)^{2}=\left(2 / N_{f}\right)\left(32 \pi / g^{2}\right)$. This equation makes sense only in the vicinity of the Fermi surface $|k-\mu| \ll \delta$ and coincides to the one derived in Ref. [9].

In the weak-coupling regime $(g /(3 \sqrt{2} \pi) \ll 1)$, the approximate solution of this equation is known to be [9]

$$
\Delta_{+}(k \sim \mu) \cong \Delta_{+}(\mu) \cos \left(\frac{g}{3 \sqrt{2} \pi} \ln \frac{|k-\mu|+\epsilon_{k}^{+}}{\Delta_{+}(\mu)}\right), \quad \Delta_{+}(\mu) \cong 2 b \mu e^{-\left(3 \pi^{2} / \sqrt{2}\right) / g},
$$

where $\epsilon_{k}^{+} \cong\left(|k-\mu|^{2}+\Delta_{+}^{2}(\mu)\right)^{1 / 2}$. This solution is a symmetric function of $(k-\mu)$ and decreases as $|k-\mu|$ increases. Inclusion of the color-electric interaction only modifies the prefactor as $b \rightarrow\left(2 / N_{f}\right)^{5 / 2} 256 \pi^{4} / g^{5}$, which enhances the gap in the weak coupling. The characteristic form of the gap $e^{-c / g}$ in Eq. (2.9), which is not the BCS form $e^{-c / g^{2}}$, was first derived in Ref. [10].

\section{Gap far from the Fermi surface in weak coupling limit}

Next, we examine the behavior of the gap far from the Fermi surface. When $k \gg \mu$, the gap equation (2.7) can be cast into the following differential equation

$$
k \frac{d^{2}}{d k^{2}} \Delta_{+}(k)+3 \frac{d}{d k} \Delta_{+}(k)+\frac{g^{2}}{3 \pi^{2}} \frac{1}{k} \Delta_{+}(k)=0,
$$

where we have assumed that $\Delta_{+}(k) \rightarrow 0$ as $k \rightarrow \infty$. The asymptotic solution of this equation for $g^{2}<3 \pi^{2}$ is

$$
\Delta_{+}(k \gg \mu) \propto k^{-\lambda}, \quad \lambda=1+\sqrt{1-g^{2} / 3 \pi^{2}} .
$$

Therefore, in the weak coupling $g \ll 1$, the gap decreases slowly as $1 / k^{2}$ for $k \gg \mu$ and does not vanish for arbitrary large $k$. This long tail is a direct consequence of the gluonic interaction which allows scattering of quarks with an arbitrary energy-momentum transfer. Otherwise, the gap cannot take the nonzero value far away from the Fermi surface.

This is in sharp contrast to the gap in the standard weak-coupling BCS superconductivity in metals, 円 where

\footnotetext{
${ }^{1}$ This is a solution of the simplest gap equation discussed in the original BCS paper 13

$$
\Delta^{\mathrm{BCS}}(k)=\frac{1}{2} \sum_{k^{\prime}} V_{k, k^{\prime}} \frac{\Delta^{\mathrm{BCS}}\left(k^{\prime}\right)}{\sqrt{\left(\epsilon_{k}-\mu\right)^{2}+\Delta^{\mathrm{BCS}}\left(k^{\prime}\right)^{2}}}
$$
}

where $V_{k, k^{\prime}} \neq 0$ for $\epsilon_{\mathrm{F}}-\omega_{\mathrm{D}}<\epsilon_{k, k^{\prime}}<\epsilon_{\mathrm{F}}+\omega_{\mathrm{D}}$ and $V_{k, k^{\prime}}=0$, otherwise. 


$$
\Delta^{\mathrm{BCS}}(p)=\left\{\begin{array}{cl}
\Delta & \left|\epsilon_{p}-\epsilon_{\mathrm{F}}\right|<\omega_{\mathrm{D}} \\
0 & \text { otherwise }
\end{array}\right.
$$

with $\epsilon_{p}, \epsilon_{\mathrm{F}}$ and $\omega_{\mathrm{D}}$ being the electron energy, the electron Fermi energy and the ultraviolet Debye cutoff of the phonon interaction originating from the lattice structure, respectively. As we mentioned above, the long tail in Eq. (2.11) is intimately related to the absence of the intrinsic ultraviolet-cutoff such as $\omega_{\mathrm{D}}$ in QCD.

\section{Full gap equation}

Now let us derive the full gap equation with momentum dependence from the original gap equation $(2.6)$ with 4 momentum dependence.

In Eq. (2.6), we adopt the static gluon propagator with the Debye screening (for the electric part) and the Landau damping (for the magnetic part). The imaginary part of the gap function will be neglected as we stated before. Using the approximation $P_{\mu \nu}^{\mathrm{L}} \approx-\delta_{\mu 0} \delta_{\nu 0}$ and performing the $q_{0}$ and angular integration, the gap equation becomes

$$
\Delta_{ \pm}(k)=\int_{0}^{\infty} d q V_{ \pm}\left(q, k ; \epsilon_{q}^{+}, \epsilon_{k}^{ \pm}\right) \frac{\Delta_{+}(q)}{\sqrt{E_{+}(q)^{2}+\left|\Delta_{+}(q)\right|^{2}}}+\int_{0}^{\infty} d q V_{\mp}\left(q, k ; \epsilon_{q}^{-}, \epsilon_{k}^{ \pm}\right) \frac{\Delta_{-}(q)}{\sqrt{E_{-}(q)^{2}+\left|\Delta_{-}(q)\right|^{2}}},
$$

where we used a simplified notation: $\Delta_{ \pm}\left(\epsilon_{k}^{ \pm}, k\right) \rightarrow \Delta_{ \pm}(k)$, and $V_{ \pm}$is defined by

$$
\begin{aligned}
V_{ \pm}\left(q, k ; q_{0}, k_{0}\right) & =\frac{g^{2}(q, k)}{48 \pi^{2}} \frac{q}{k}\left[\frac{1}{3} \ln \left(\frac{(k+q)^{6}+M^{4}\left(q_{0}-k_{0}\right)^{2}}{(k-q)^{6}+M^{4}\left(q_{0}-k_{0}\right)^{2}}\right)+\left(q_{0} \rightarrow-q_{0}\right)+f_{\mathrm{MG}}\right] \\
& +\frac{g^{2}(q, k)}{48 \pi^{2}} \frac{q}{k}\left[\frac{(k \pm q)^{2}+m_{\mathrm{D}}^{2}}{2 q k} \ln \left(\frac{(k+q)^{2}+m_{\mathrm{D}}^{2}}{(k-q)^{2}+m_{\mathrm{D}}^{2}}\right)+f_{\mathrm{EL}}\right] .
\end{aligned}
$$

Here $f_{\mathrm{EL}}$ is just a constant, and $f_{\mathrm{MG}}$ is a rather complicated function of $q$ and $k$. Since no singularity appears in $f_{\mathrm{EL}, \mathrm{MG}}$ when $m_{\mathrm{D}}=0, M=0$ and $q=k=\mu$ are taken, they are considered to be sub-leading contribution and will be neglected in the following analysis. In the definition of $V_{ \pm}$, the first (second) term corresponds to the magnetic (electric) gluon contribution. We call the first integral in Eq. (2.13) as "quark-pole contribution" and the second integral as "antiquark-pole contribution", because $E_{+}=q-\mu\left(E_{-}=q+\mu\right)$ is involved in the former (latter). Near the Fermi surface $q \sim \mu$, the first integral in Eq. (2.13) gives the dominant contribution due to the small denominator $\left(E_{+}^{2}+\left|\Delta_{+}\right|^{2}\right)^{1 / 2}=\left\{(q-\mu)^{2}+\left|\Delta_{+}\right|^{2}\right\}^{1 / 2} \sim 0$.

At extremely high density, the Cooper pairing is expected to take place only near the Fermi surface as we have discussed. In this case, we can safely neglect the antiquark-pole contribution for calculating $\Delta_{+}$. Furthermore, one may replace the momentum dependent vertex by the coupling constant on the Fermi surface $g^{2}(q, k) \rightarrow g^{2}(q=$ $\mu, k=\mu)$. If $\mu$ is large enough, $g^{2}(q=\mu, k=\mu)$ may be identified with the standard running coupling $g^{2}(\mu)=$ $\left(8 \pi^{2} / \beta_{0}\right) / \ln \left(\mu^{2} / \Lambda_{\mathrm{QCD}}^{2}\right)$. Thus, keeping only the magnetic interaction, one recovers the gap equation (2.7) for $\Delta_{+}$with $g^{2} \rightarrow g^{2}(\mu)$ in the weak-coupling limit.

At low densities, sizable diffusion of the Fermi surface occurs and the weak-coupling approximations leading to Eq. (2.7) are not justified. Therefore, we need to solve the coupled gap equations Eq. (2.13) numerically. In particular, the replacement $g^{2}(q, k) \rightarrow g^{2}(q=\mu, k=\mu)$ is not justified when $q$ and $k$ are not close to $\mu$. The contribution from the antiquark-pole is also not entirely negligible.

\section{Occupation number, correlation function and coherence length}

To clarify the structural change of the color superconductor from high to low densities, it is useful to examine the following physical quantities related to the gap function.

(i) The quark and antiquark occupation number in each momentum state: This is a fundamental quantity characterizing the diffuseness of the Fermi surface. It is related to the diagonal (1-1) element of the quark propagator in the Nambu-Gor'kov formalism

$$
\begin{aligned}
& \left\langle\psi_{j}^{\dagger b}(t, \boldsymbol{y}) \psi_{i}^{a}(t, \boldsymbol{x})\right\rangle_{\text {super }}=\lim _{x^{0} \rightarrow y^{0}-\epsilon}\left(-i\left[S_{11}\right]_{i j}^{a b}(x-y) \gamma^{0}\right) \\
& \quad=\int \frac{d^{3} \boldsymbol{q}}{(2 \pi)^{3}} e^{i \boldsymbol{q}(\boldsymbol{x}-\boldsymbol{y})}\left[\left\{\Lambda_{+}(\hat{\boldsymbol{q}}) \theta(\mu-|\boldsymbol{q}|)+\Lambda_{-}(\hat{\boldsymbol{q}})\right\}\left(P_{3}^{c}\right)_{a b}\left(\mathbf{1}_{\mathrm{F}}\right)_{i j}\right.
\end{aligned}
$$




$$
\left.+\left\{\frac{1}{2}\left(1-\frac{E_{+}(q)}{\sqrt{E_{+}(q)^{2}+\left|\Delta_{+}(q)\right|^{2}}}\right) \Lambda_{+}+\frac{1}{2}\left(1+\frac{E_{-}(q)}{\sqrt{E_{-}(q)^{2}+\left|\Delta_{-}(q)\right|^{2}}}\right) \Lambda_{-}\right\}\left(1-P_{3}^{c}\right)_{a b}\left(\mathbf{1}_{\mathrm{F}}\right)_{i j}\right],
$$

where $P_{3}^{c}$ is a projection matrix to the third axis in the color space. From this expression, one can extract the quark and the antiquark occupation numbers as

$$
n_{ \pm}^{1,2}(q)=\frac{1}{2}\left(1-\frac{E_{ \pm}(q)}{\sqrt{E_{ \pm}(q)^{2}+\left|\Delta_{ \pm}(q)\right|^{2}}}\right), \quad n_{+}^{3}(q)=\theta(\mu-q), \quad n_{-}^{3}(q)=0
$$

where the superscripts (1,2 and 3) stand for color indices. Since the third axis in the color space is chosen to break the color symmetry, quarks with the third color do not contribute to form Cooper pairs. When the gap is zero $\Delta_{ \pm}=0$, the system reduces to the ordinary quark matter with a sharp Fermi sphere; $n_{+}^{1,2}(q)=\theta(\mu-q)$ and $n_{-}^{1,2}(q)=0$.

(ii) The $q-q$ and $\bar{q}-\bar{q}$ correlation functions in the momentum space $\hat{\varphi}_{ \pm}(q)$ and in the coordinate space $\varphi_{ \pm}(r)$ : They reflect the internal structure of the Cooper pairs in color superconductor. These correlations are related to the off-diagonal (1-2) element of the quark propagator:

$$
\begin{aligned}
& \left\langle\psi_{i}^{a}(t, \boldsymbol{x}) \psi_{j}^{b}(t, \boldsymbol{y})\right\rangle_{\text {super }}=\lim _{x^{0} \rightarrow y^{0}+\epsilon} i\left[S_{12}\right]_{i j}^{a b}(x-y) \\
& =\int \frac{d^{3} \boldsymbol{q}}{(2 \pi)^{3}} e^{i \boldsymbol{q}(\boldsymbol{x}-\boldsymbol{y})}\left(\frac{\Lambda_{+}(\hat{\boldsymbol{q}}) \Delta_{+}(q)}{2 \sqrt{E_{+}(q)^{2}+\left|\Delta_{+}(q)\right|^{2}}}+\frac{\Lambda_{-}(\hat{\boldsymbol{q}}) \Delta_{-}(q)}{2 \sqrt{E_{-}(q)^{2}+\left|\Delta_{-}(q)\right|^{2}}}\right)\left(i \gamma_{5} C\right)\left(\lambda_{2} \tau_{2}\right)_{i j}^{a b} .
\end{aligned}
$$

$\hat{\varphi}_{ \pm}(q)$ is simply extracted from the above and $\varphi_{ \pm}(r)$ is defined as the Fourier transform

$$
\hat{\varphi}_{ \pm}(q)=\frac{\Delta_{ \pm}(q)}{2 \sqrt{E_{ \pm}(q)^{2}+\left|\Delta_{ \pm}(q)\right|^{2}}}, \quad \varphi_{ \pm}(r)=N \int \frac{d^{3} \boldsymbol{q}}{(2 \pi)^{3}} \hat{\varphi}_{ \pm}(q) e^{i \boldsymbol{q} \boldsymbol{r}}
$$

where $N$ is a normalization constant determined by $\int d^{3} r\left|\varphi_{+}(r)\right|^{2}=1$.

(iii) The coherence length $\xi_{\mathrm{c}}$ characterizing the typical size of a Cooper pair: It is defined simply as a root mean square radius of $\varphi_{+}(r)$ :

$$
\xi_{\mathrm{c}}^{2}=\frac{\int d^{3} r r^{2}\left|\varphi_{+}(r)\right|^{2}}{\int d^{3} r\left|\varphi_{+}(r)\right|^{2}}=\frac{\int_{0}^{\infty} d k k^{2}\left|d \hat{\varphi}_{+}(k) / d k\right|^{2}}{\int_{0}^{\infty} d k k^{2}\left|\hat{\varphi}_{+}(k)\right|^{2}}
$$

A measure of the coherence length $\xi_{\mathrm{c}}$ in the weak-coupling limit is known as the Pippard length, which is given by $\xi_{\mathrm{p}}=\left(\pi \Delta_{+}(\mu)\right)^{-1}[25]$. It is shown in Appendix B that the quark correlation $\varphi_{+}(r)$ in the weak-coupling limit behaves as

$$
\varphi_{+}(r \rightarrow \infty) \propto \frac{\sin (\mu r)}{(\mu r)^{3 / 2}} \cdot e^{-r /\left(\pi \xi_{\mathrm{p}}\right)}
$$

In a typical type-I superconductor in metals, the Pippard length is of the semi-macroscopic order $\xi_{\mathrm{p}} \sim 10^{-4} \mathrm{~cm}$, whereas inverse Fermi momentum is of the microscopic order $k_{\mathrm{F}}^{-1} \sim 10^{-8} \mathrm{~cm}$. The inverse of the Debye cutoff is in between the two scales $\omega_{\mathrm{D}}^{-1} \sim 10^{-6} \mathrm{~cm}$. Therefore there is a clear scale hierarchy, $\Delta \ll \omega_{\mathrm{D}} \ll k_{\mathrm{F}}$. Because of the absence of the intrinsic scale $\omega_{\mathrm{D}}$, similar scale hierarchy in QCD at extremely high density reads $\mu e^{-c / g} \ll \mu$. At lower densities, however, such scale separation becomes questionable for $g$ is not small.

\section{NUMERICAL RESULTS}

In this section, we present numerical results of the momentum-dependent gap and the other physical quantities. In Sect. IIIA, we show solutions of the gap equations at very high density. Then we discuss whether the result has similarity to that in the BCS superconductivity for metals [see Eq. (2.12)]. What makes the color superconductor unique is the absence of the intrinsic cutoff scale $\omega_{\mathrm{D}}$. Nevertheless, we will see that similar relation as Eq. (2.12) holds under the replacement $\omega_{\mathrm{D}} \rightarrow \Delta$ at least at extremely high density. We will also examine how the results of the gap equation in the weak-coupling limit (2.7) are modified when the effects such as the color-electric interaction, the momentum dependent coupling and the antiquark-pole contribution are taken into account. 
In Sects. IIIB and IIIC, we repeat the same calculations at lower densities and show a qualitative difference from the weak-coupling limit. Substantial modification of the Fermi surface at low densities will be explicitly shown by computing the occupation number. Quark correlation in the color superconductor and the size of the Cooper pair are calculated in Sect. IIID. The results indicate that the color superconductivity at low densities is no longer similar to the usual BCS-type superconductivity.

In Sects. IIIA and IIIB, starting from the simplest gap equation in the weak-coupling limit (2.7), we will include the contributions of color-electric interaction, momentum-dependent coupling and antiquark pole, step by step. This procedure clarifies the importance of each contribution. Let us define each step below, for later convenience.

Step 1: We solve the gap equation for $\Delta_{+}$in the weak-coupling limit Eq. (2.7) where only the color-magnetic interaction is taken into account. The momentum independent coupling $g^{2}(\mu, \mu)$ is used in this step.

Step 2: The Debye-screened color-electric interaction is further added to Step 1. This corresponds to solving Eq. (2.13) for $\Delta_{+}$with neglecting the antiquark-pole contribution and with making a replacement $g^{2}(q, k) \rightarrow$ $g^{2}(\mu, \mu)$.

Step 3: Same as Step 2 except for the use of $g^{2}(q, k)$ instead of $g^{2}(\mu, \mu)$.

Step 4: The antiquark-pole contribution is also taken into account in Step 3. This gives the complete solution of our full coupled gap equations Eq. (2.13).

Throughout the all steps, $g^{2}$ in the Debye screening mass $m_{\mathrm{D}}$ is taken to be $g^{2}(\mu, \mu)$ for simplicity. In Step 3 and Step 4 , we use a phenomenological value $\Lambda=400 \mathrm{MeV}$ and $p_{c}^{2}=1.5 \Lambda^{2}$ for $g(q, k)$ as we have already mentioned in Sect. IIA.

\section{A. Momentum dependence of the gap at high density}

In this subsection, we solve the gap equations at chemical potential $\mu=2^{12} \Lambda \simeq 1.6 \mathrm{TeV}$. This corresponds to the baryon density $\rho_{\mathrm{B}}=1.1 \times 10^{11} \rho_{0}$ with $\rho_{0}=0.17 \mathrm{fm}^{-3}$ being the normal nuclear matter density. At this extremely high density, we expect that the full gap equation Eq. (2.13) is well approximated by its weak-coupling limit, Eq. (2.7). Furthermore, the analytic solution Eq. (2.9) is expected to give a fair approximation of Eq. (2.7) around the Fermi surface. In Fig. 1, we show the numerical results of the four steps. It is evident that all the results have a very narrow peak at the Fermi surface. This peak is not a singular one, but has a plateau with the width of $2 \Delta_{+}(k=\mu)$, as we will show later. At high momentum $k \gg \mu$, the decrease of the gap obeys the power law. This is consistent with the analytic solution Eq. (2.11). Such a long tail is due to the unscreened nature of the color-magnetic interaction and is quite different from the behavior of the gap in the standard BCS-type superconductivity. Let us look into each step in more detail.

\section{Steps 1 and 2:}

Comparing the gap with the magnetic interaction alone (the dotted line) and the gap with both magnetic and electric interactions (the dashed line), we find that the electric interaction induces a large enhancement (about 10 times) of the gap almost independently of momentum. This is already suggested by the analytic studies in Sect. IIC.

This enhancement may be understood in a qualitative manner. In the coordinate space, the Debye-screened electric interaction behaves as a Yukawa potential. Such a short-range interaction can form only a loosely bound Cooper pairs with a large size. In fact, if one solves the gap equation with the electric interaction alone, one finds a very small gap compared to the one in Fig. 2(a). The situation becomes different when the magnetic and electric interactions coexist: Small size Cooper pairs are formed primarily by the long-range magnetic interaction. Then, even the short-range electric interaction becomes effective to generate further attraction between the quarks. This cooperative effect can qualitatively explain the reason why addition of the electric interaction enhances the gap.

\section{Steps 3 and 4:}

To see the effect of the momentum dependent coupling $g^{2}(q, k)$, let us compare the dot-dashed line (where $g^{2}(q, k)$ is used) with the dashed line (where $g^{2}(\mu, \mu)$ is used). Since $g^{2}(q, k)$ works as a weight factor in the momentum integral in the gap equation, substantial difference should appear between the two cases if the contribution away from the Fermi surface is not negligible in the integral. However, we find no significant difference between the two cases at high density. This implies that the color superconductivity at high density is governed by the physics near the Fermi surface. 
Let us examine the last stage, Step 4, where the antiquark-pole contribution is included. The solid line in Fig. 11 is $\Delta_{+}(k)$ obtained by solving the full coupled gap-equation (2.13) for $\Delta_{+}$and $\Delta_{-}$. Since the difference from the previous step is small, we can conclude that the quark-pole dominance is indeed a good approximation at very high density. The antiquark gap $\Delta_{-}(k)$ is shown in a separate figure (Fig. 2) together with $\Delta_{+}(k)$ (the same as the solid line in Fig. 1). Unlike $\Delta_{+}(k)$, the antiquark gap $\Delta_{-}(k)$ is a smoothly decreasing function of the momentum. Although $\Delta_{-}$ is not small compared to $\Delta_{+}$, it does not imply that the sizable antiquark Cooper pairs exist in the system because the number of antiquarks are much smaller than quarks as we will show in Sect. IIID.

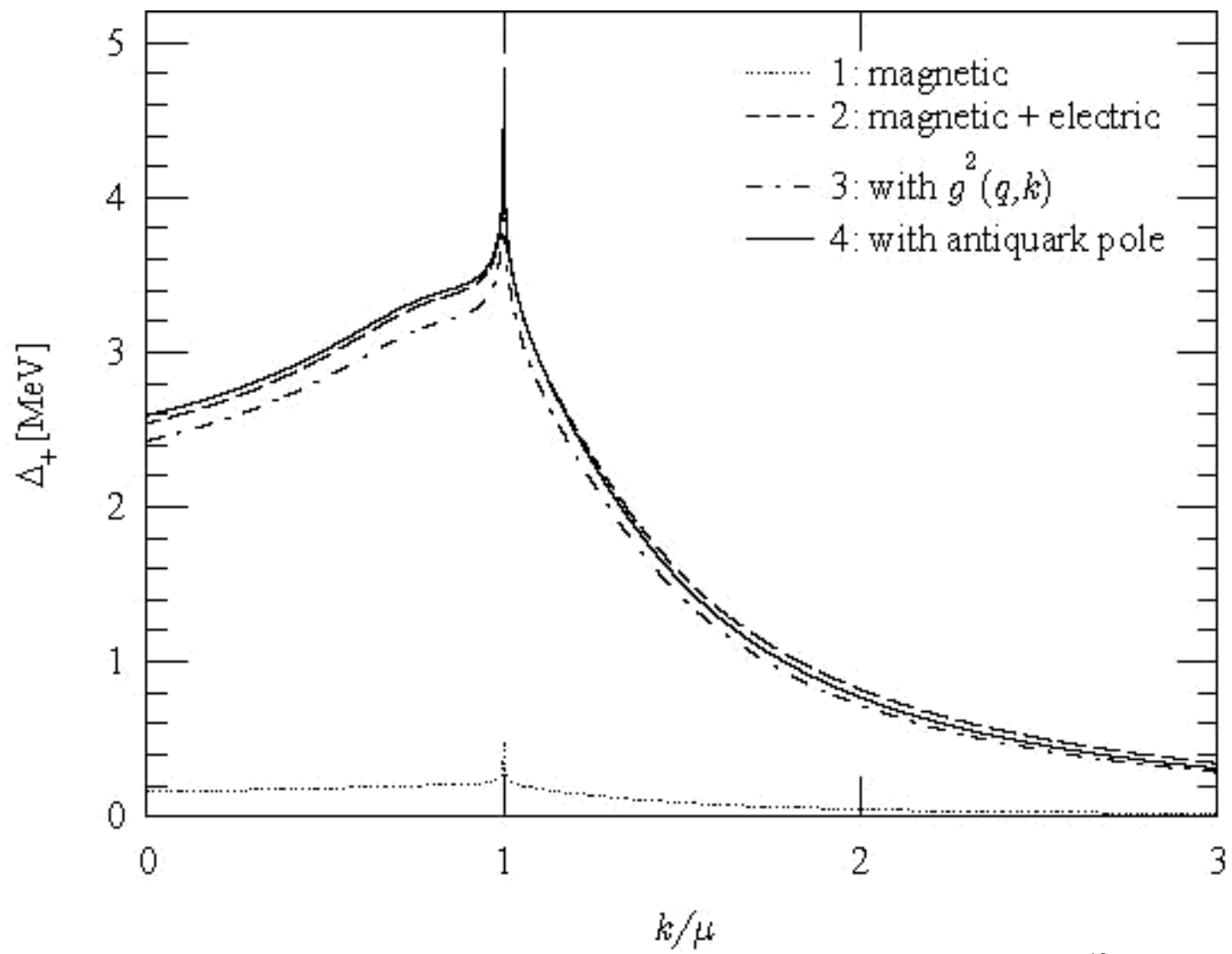

FIG. 1. The momentum dependence of the gap $\Delta_{+}$from Step 1 to Step 4 at high density, $\mu=2^{12} \Lambda$. The dotted line is a result of the magnetic interaction alone (Step1). The dashed line is the gap with both magnetic and electric-interactions included (Step2). The dot-dashed line is the gap in which the momentum dependent coupling $g^{2}(q, k)$ is used (Step 3) instead of the momentum independent coupling $g^{2}(\mu, \mu)$ (Step 2). The solid line is the gap as a solution of the full gap equation (2.13) with $g^{2}(q, k)$ and the antiquark pole (Step 4).

Finally, Fig. 3 shows a comparison between the momentum dependence of the numerical solution $\Delta_{+}$in Step 4 (the solid line which is the same as the solid line in Fig. 2) with the analytic solution near the Fermi surface (2.9) (the dashed line). The peak height of the analytic solution is adjusted so that the solid and dashed lines coincide at $k=\mu$. In the vicinity of the Fermi surface (Fig. 3(a)), the momentum dependence of the analytic solution agrees with the numerical solution reasonably well. Note that the peak is not a singular one as we mentioned before. On the other hand, as is shown in Fig. 3(b) the deviation becomes considerable away from the Fermi surface. In particular, the asymmetry with respect to the Fermi surface $k=\mu$ represents the deviation from weak coupling analytic solution. Note that the coincidence at $k \sim 0$ is accidental because the analytic result (2.9) is valid only near the Fermi surface.

In this subsection, we have solved the momentum-dependent gap equations at high density $\mu=2^{12} \Lambda$. The characteristic features of the momentum-dependent gap are (i) there is a sharp peak at the Fermi surface, and (ii) the gap decays rapidly but is nonzero for momentum far away from the Fermi surface. The property (i) is similar to the standard BCS superconductivity [see Eq. (2.12]] but (ii) is not, due to the absence of intrinsic ultraviolet Debye-cutoff of the gluonic interaction in QCD. As for the magnitude of the gap at high density, the color-electric interaction enhances the gap considerably. The effects of the momentum-dependent coupling $g^{2}(q, k)$ and the antiquark pairing are shown to be not important at high density. 


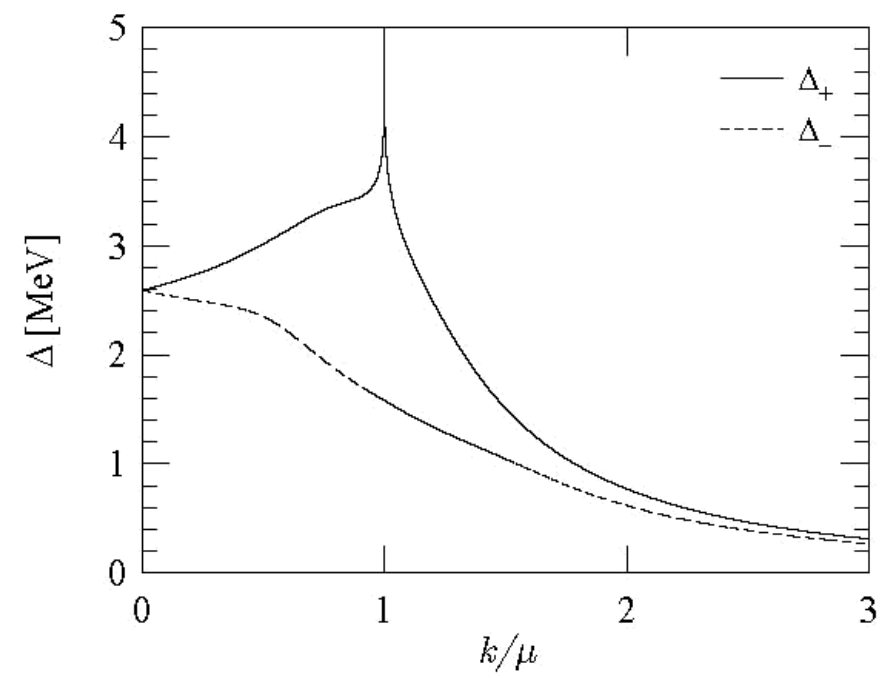

FIG. 2. $\Delta_{+}$and $\Delta_{-}$as the solutions to the full coupled gap-equation in Step 4 at high density, $\mu=2^{12} \Lambda$.

(a)

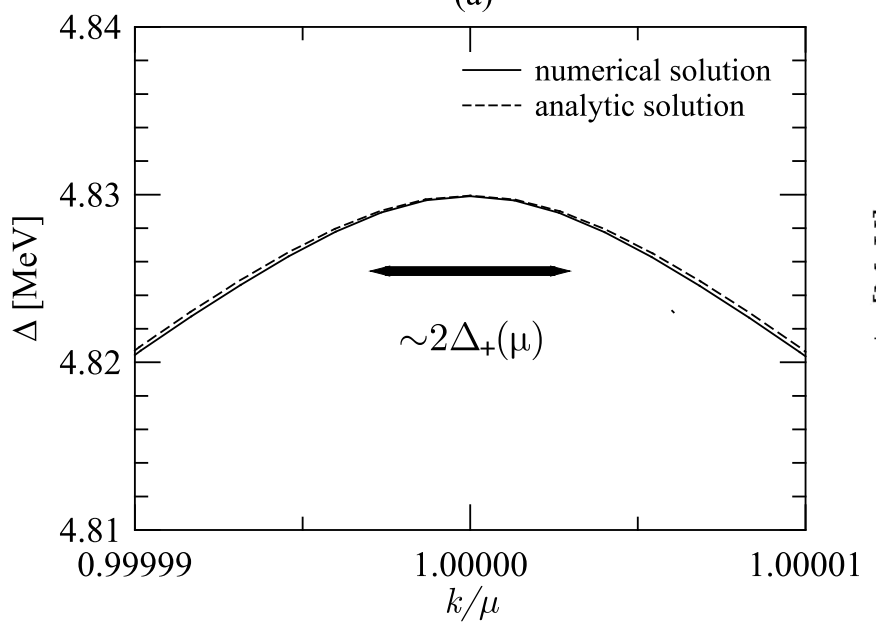

(b)

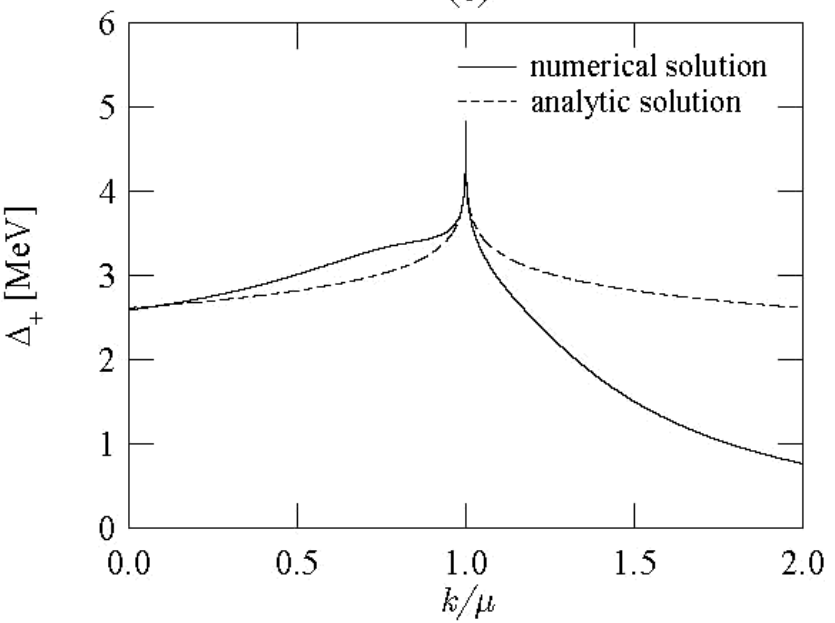

FIG. 3. Comparison of the numerical solution of $\Delta_{+}$in Step 4 (the solid line) and the analytic solution (2.9) (the dashed line) at high density, $\mu=2^{12} \Lambda$. The latter is normalized to the former at $k=\mu$. (a) near the Fermi surface, (b) global behavior.

\section{B. Momentum dependence of the gap at low density}

In this subsection, we carry out the same calculations as in Sect. IIIA at lower density $\mu=2 \Lambda=800 \mathrm{MeV}$ (which corresponds to the baryon density $\rho_{\mathrm{B}}=13.2 \rho_{0}$ ). In Fig. 囵, we show the numerical results of the four steps. One of the main differences from the high density case is the absence of a sharp peak. The width of the peak in Fig. 1 in terms of $k / \mu$ is much wider than that in Fig. 1. This large modification of the gap function will be attributed to the larger coupling at low density, because the Cooper pairs far from the Fermi surface are easily formed. Therefore, this implies that the physics of superconductivity is no longer limited on the Fermi surface. We confirm this below by more detailed analysis of each steps. 


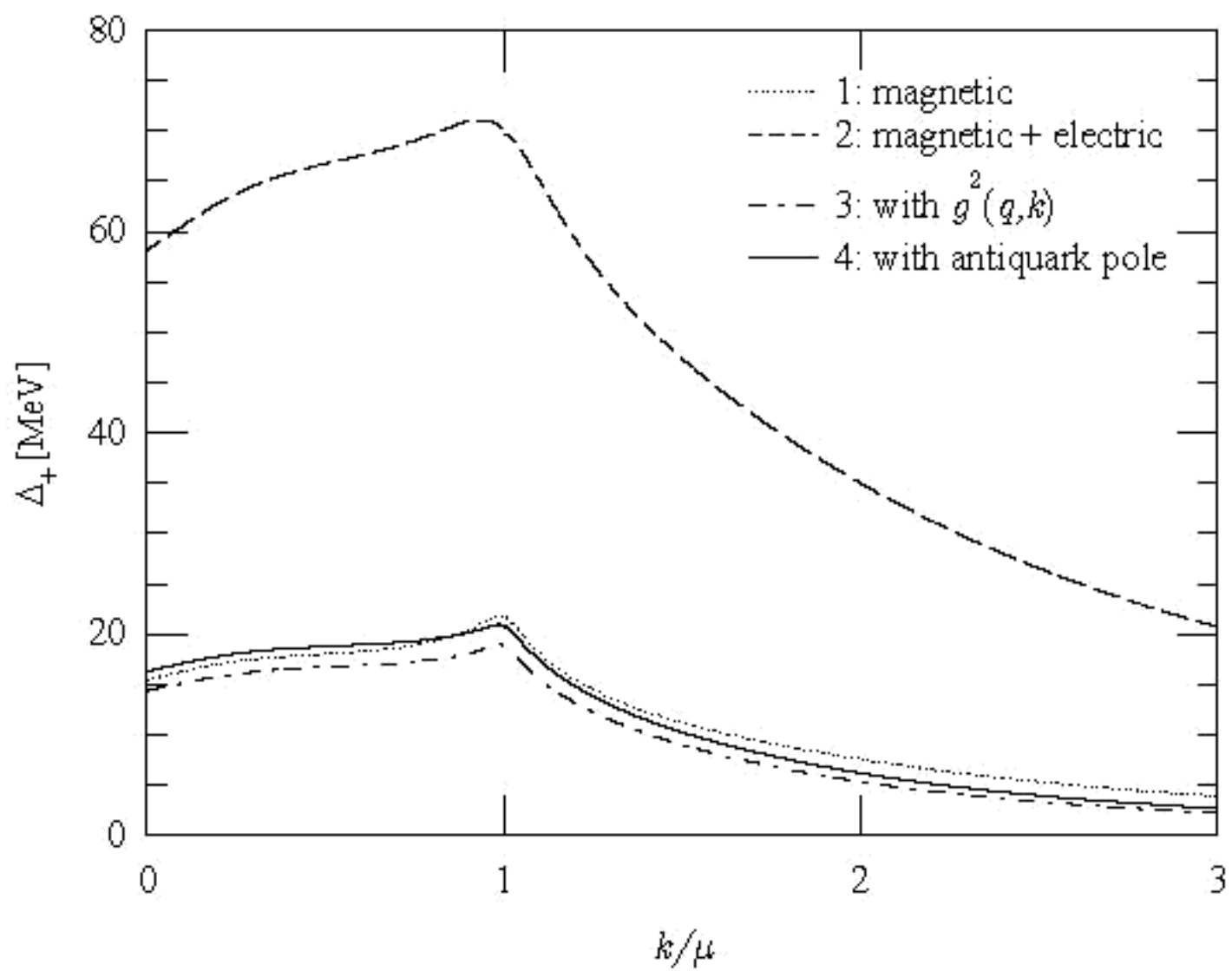

FIG. 4. Same as Fig. 1 except that the density is lower, $\mu=2 \Lambda=800 \mathrm{MeV}$.

\section{Steps 1 and 2:}

Comparing the dotted line (with the magnetic interaction alone) and the dashed line (magnetic and electric interactions) in Fig. (1, we again find that the addition of the color-electric interaction induces a large enhancement of the gap.

\section{Steps 3 and 4:}

The result of Step 3 (where $g^{2}(q, k)$ is used) is shown as the dot-dashed line, which should be compared with the previous step (the dashed line) where $g^{2}(\mu, \mu)$ is used. In contrast to the high density case in Fig. 1, the momentum dependent coupling makes the magnitude of the gap considerably smaller. This is one of the evidences that the momentum integration is not any more dominated by the small region near the Fermi surface. The reason why the suppression of the gap takes place instead of the enhancement is understood as follows. Consider the gap at the Fermi surface $\Delta_{+}(k=\mu)$. In the momentum integration of the gap equation Eq. (2.13) at $k=\mu, g^{2}(q, k=\mu)<g^{2}(\mu, \mu)$ for $\mu<q$, while $g^{2}(q, k=\mu)=g^{2}(\mu, \mu)$ for $\mu>q$. Therefore, the momentum-dependent coupling always acts to reduce the magnitude of the integral as compared to the momentum-independent one.

Now, let us include the antiquark excitation in the gap equation (Step 4). The solution of our full gap equation (2.13) is shown by the solid line. The antiquark-pole contribution enhances the gap by $10 \%$. The difference between the solid and dot-dashed lines is not huge but the absolute magnitude of the enhancement is larger than that in the extremely high density case shown in Fig. 1. The reason for this enhancement is clear: As one decreases density, the chemical potential becomes small, which plays a role of the intrinsic energy gap for antiquark excitation. Therefore, the antiquark-pole contribution becomes non-negligible. This also suggests that the more low-momentum antiquarks are present at low density which will be confirmed in Sect. IIID by computing the occupation number of antiquarks.

Finally, in Fig. 周, we compare the quark-gap $\Delta_{+}$(the solid line) and the antiquark gap $\Delta_{-}$(the dashed line). As in the case of high density, the antiquark gap $\Delta_{-}$is a smooth function of $k$ and is not small compared to $\Delta_{+}$.

In this subsection, we found that the momentum dependence of the gap at low density is quite different from that at very high density. The sharp peak at the Fermi surface disappears. The gap equation in the weak-coupling limit (2.7) 
is no longer a good approximation and all the contributions neglected in the weak-coupling limit are not negligible. All these results allow us to conclude that the color superconductivity at low density is not a phenomenon just around the Fermi surface. In subsections IIID and IIIE below, we will strengthen this picture by looking at other quantities such as the quark occupation numbers and the size of the Cooper pair.

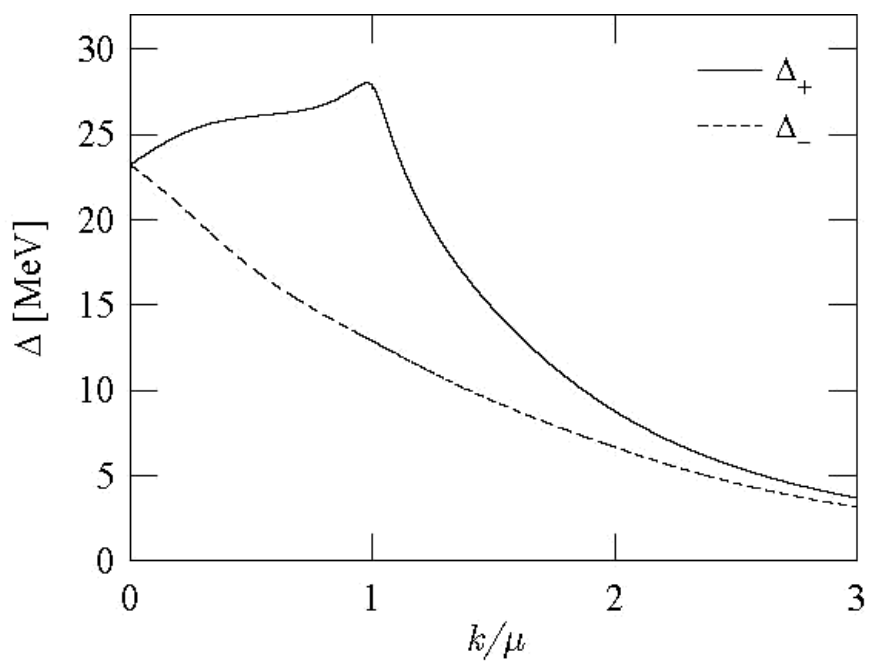

FIG. 5. Same as Fig. 2 except that the density is lower, $\mu=2 \Lambda=800 \mathrm{MeV}$.

\section{Density dependence of the gap}

Let us now examine how the gap at high density with a sharp peak changes into the gap at low density with only a broad bump. In Fig. 6(a), we show the gap $\Delta_{+}(k)$ as a solution of the full gap equation (Step 4) for a wide range of densities. Since the actual position of the Fermi surface moves as we vary the density, we use $k / \mu$ as a horizontal axis in the figure in order to show the change of the global behavior. The figure shows that the sharp peak at high density gradually gets broadened and simultaneously the magnitude of the gap increases as we decrease the density.

In Fig. 6(b), the gap at the Fermi surface $\Delta_{+}(\mu)$ is shown as a function of the chemical potential. It decreases monotonically as $\mu$ increases, but turns into an increase for $\mu>10^{6} \mathrm{MeV}$. The analytic solution is also shown in Fig. 6(b). The magnitude of the analytic solution is normalized to the numerical solution at the highest density $\mu=2^{12} \Lambda \simeq 1.6 \times 10^{6} \mathrm{MeV}$. At high density, $\mu$-dependence of the numerical result is in good agreement with the analytic form which has a parametric dependence $\Delta_{+}(\mu) \propto g^{-5} \mu \exp \left(-3 \pi^{2} / \sqrt{2} g\right)$ with $g^{2}=g^{2}(\mu, \mu)$. On the other hand, the difference of the two curves at low density implies the failure of the weak-coupling approximation. As we have seen before, the use of $g^{2}(q, k)$ and the antiquark-pole has non-negligible effects on the gap in the low density regime. 
(a)

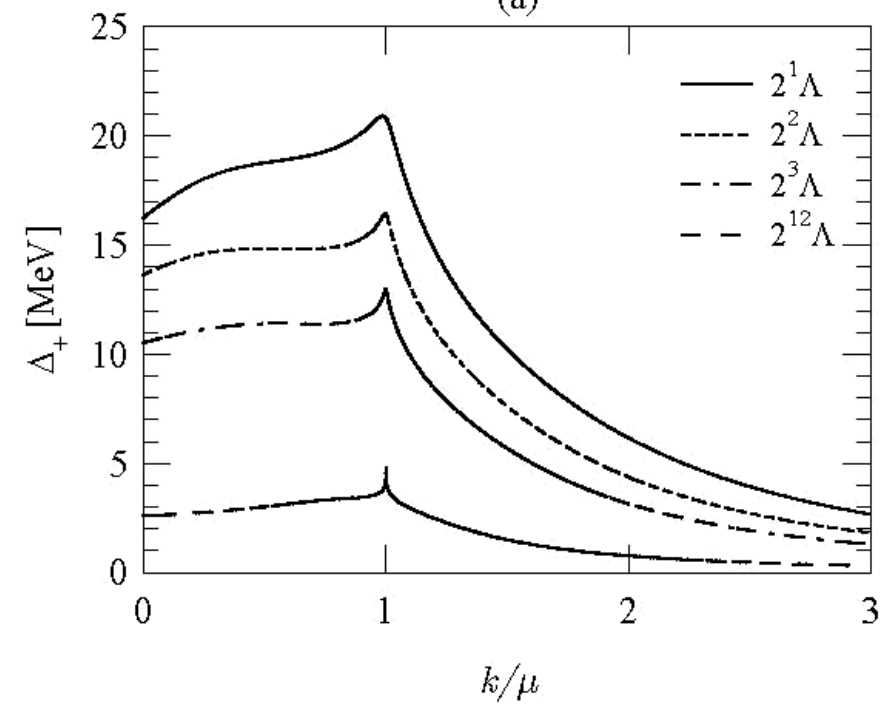

(b)

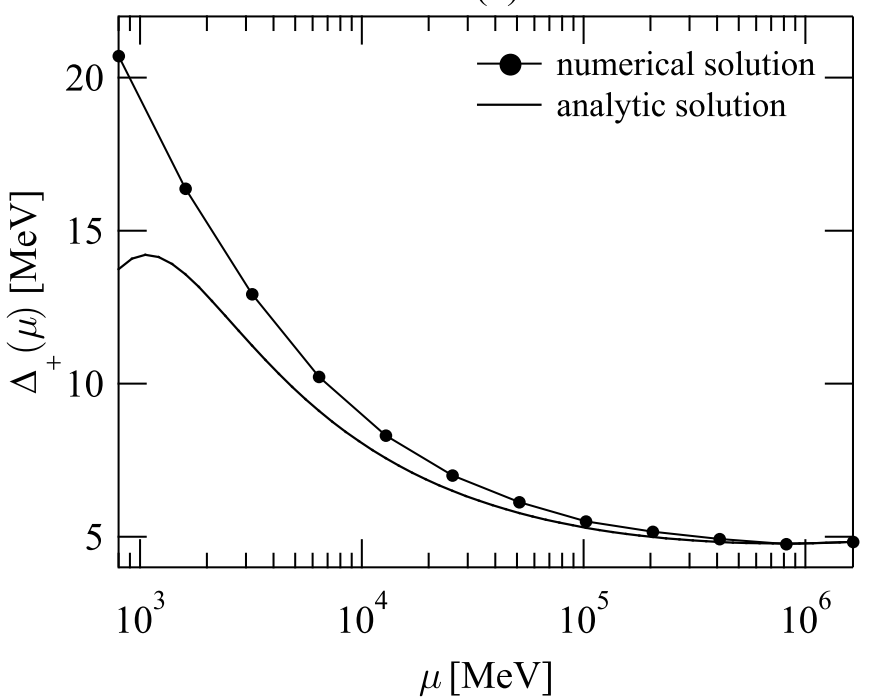

FIG. 6. (a) $\Delta_{+}(k)$ as a function of $k / \mu$ for various densities $\mu=2^{n} \Lambda$ with $n=1,2,3,12$. All the calculations are done with the momentum dependent vertex $g^{2}(q, k)$ and with the antiquark-pole contribution. (b) Chemical potential dependence of the gap $\Delta_{+}(k=\mu)$ in the full calculation compared with the analytic result which is normalized at the highest density $\mu=2^{12} \Lambda$.

\section{Occupation numbers}

So far we have seen that the weak-coupling picture of the color superconductivity is modified at low densities. In order to see how the Fermi surface is diffused by the Cooper pairing, let us evaluate the occupation numbers of quarks and antiquarks. Since we have solved the full gap equation (2.13) for $\Delta_{ \pm}(k)$, we can immediately obtain the occupation numbers using Eq. (2.16).

In Fig. 7, the occupation numbers of quarks [Fig. 7(a)] and antiquarks [Fig. 7(b)] are shown at high density $\mu=2^{12} \Lambda$ and at lower densities $\mu=2 \Lambda, 2^{2} \Lambda$. One finds that the quark occupation numbers is almost a step function at high density, while it is smeared out for a wide region of momentum at low densities. The diffuseness of the Fermi surface is found to be of the order of $\Delta_{+}(\mu)$, which is consistent with the definition in Eq. (2.16).

Figure (b) implies that a small amount of the antiquarks also participate in the color superconductivity. Although the antiquark gap $\Delta_{-}$is of the same order of the quark gap $\Delta_{+}$, the antiquark occupation number is generally suppressed due to the large energy denominator $\left\{(k+\mu)^{2}+\left|\Delta_{-}\right|^{2}\right\}^{1 / 2}$. As one decreases the density, however, such suppression is relatively weakened and the magnitude of the antiquark occupation number increases, as can be seen from Figure $7(\mathrm{~b})$.

(a)

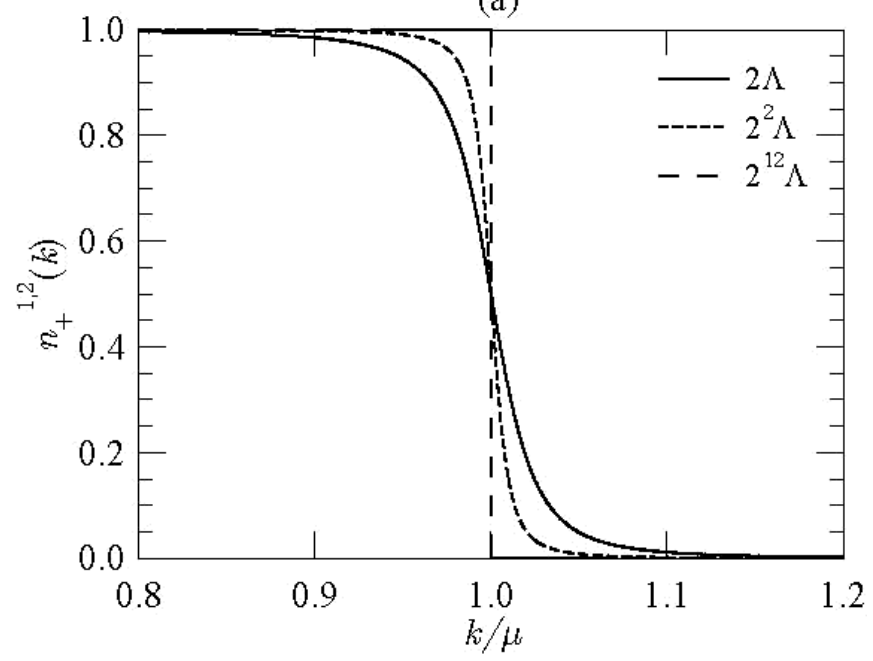

(b)

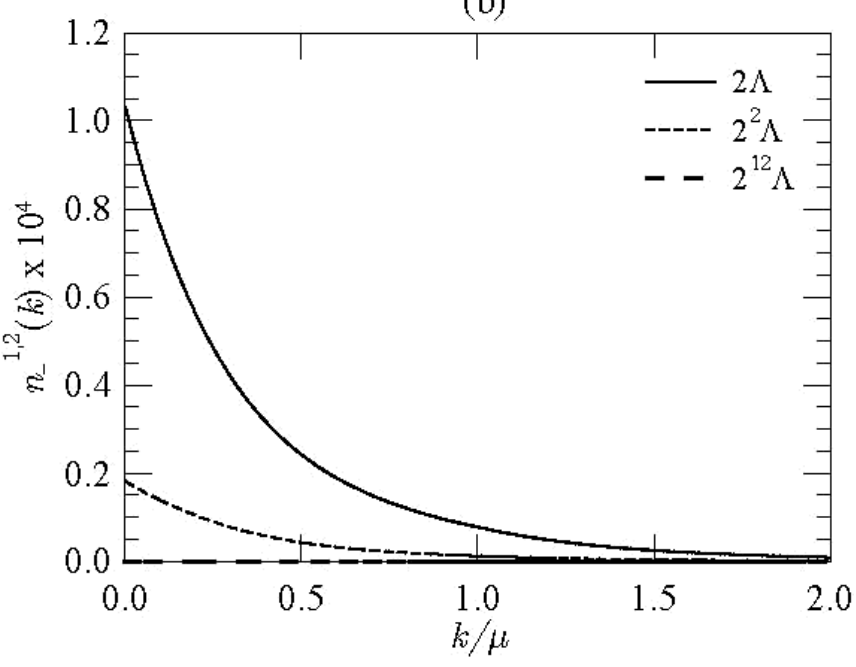


FIG. 7. Occupation numbers of quarks $n_{+}^{1,2}$ (a) and antiquarks $n_{-}^{1,2}$ (b) for several different densities.

\section{E. Correlation function and coherence length}

One of the advantages of treating the momentum dependent gap is that we are able to calculate the correlation function which physically corresponds to the "wavefunction" of the Cooper pair. Such correlations have been first studied in Ref. 15 in the context of the color superconductivity.

Using Eq. (2.18), we calculate the correlation functions of quarks and antiquarks, $\hat{\varphi}_{ \pm}(k)$. The results at various densities are shown in Fig. 8. For quarks [Fig. 8(a)], the correlation function at very high density has a sharp peak at the Fermi surface but it becomes broader as we decrease the density. This is of course due to the broadening of the gap which we found in Fig. 6 .

For antiquarks [Fig. 8(b)], the correlation is much weaker than that of quarks and is a smoothly decreasing function of $k$. Also, the magnitude of the correlation increases as we decrease the density. Since $\Delta_{-}(k) \ll \mu$ holds for densities considered in this paper, the above features can be simply understood by an approximate relation, $\hat{\varphi}_{-}(k) \sim$ $\Delta_{-}(k) / 2(k+\mu)$.

(a)

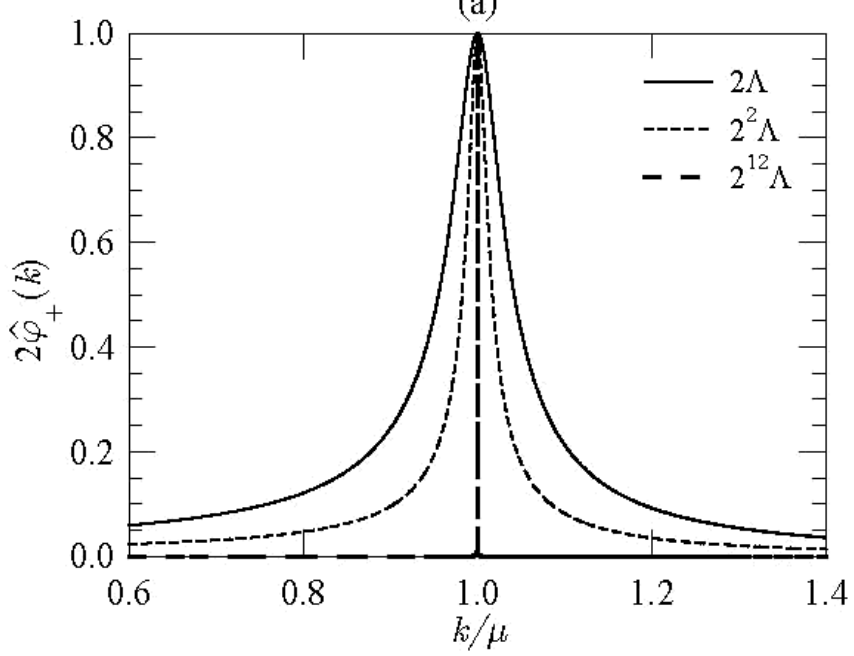

(b)

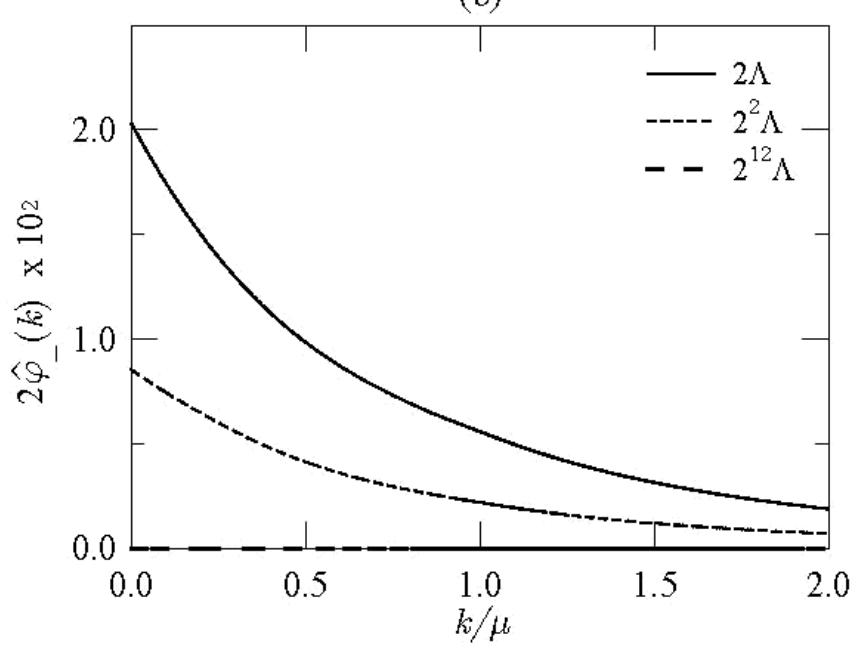

FIG. 8. The correlation functions in the momentum space at several different densities for quarks (a) and for antiquarks (b).

We can compute the size of a Cooper pair from the correlation function. Figure 9(a) shows the coherence length $\xi_{c}$ of a quark Cooper pair defined as the root mean square radius of the correlation function [see Eq. (2.19)]. The size of a Cooper pair becomes smaller as we go to lower densities. This tendency is understood by the behavior of the Pippard length $\xi_{\mathrm{p}}=1 / \pi \Delta_{+}(\mu)$ (which gives a rough estimate of the coherence length) together with the behavior of $\Delta_{+}(\mu)$ shown in Fig. $6(\mathrm{~b})$.

Also, the Cooper pair becomes smaller as density increases beyond $\mu=2^{12} \Lambda$. However, it does not necessarily imply the existence of tightly bound Cooper pairs. In fact, the size of a Cooper pair makes sense only in comparison to the typical length scale of the system, namely the averaged inter-quark distance $d_{q}$ defined as 2

$$
d_{q}=\left(\frac{\pi^{2}}{2}\right)^{1 / 3} \frac{1}{\mu}
$$

As we go to higher densities, the ratio $\xi_{\mathrm{c}} / d_{q}$ increases monotonically as shown in Fig. 9(b). Namely loosely bound Cooper pairs similar to the BCS superconductivity in metals are formed at extremely high densities.

At the lowest density in Fig. 9, the size of the Cooper pair is less than $4 \mathrm{fm}$ and the ratio $\xi_{\mathrm{c}} / d_{q}$ is less than 10. The transition from $\xi_{\mathrm{c}} / d_{q} \gg 1$ to $\xi_{\mathrm{c}} / d_{q} \sim 1$ as $\mu$ decreases is analogous to the transition from the BCS-type

\footnotetext{
${ }^{2}$ This is a result of free quarks. To obtain accurate density $\rho$ and $d_{q}$, we have to include contributions from interaction. However, such correction is suppressed by the power of $\Delta_{+} / \mu$.
} 
superconductor to the so-called "strong coupling" superconductor. The weak-coupling BCS superconductivity may smoothly change into the Bose-Einstein condensation (BEC) of tightly bound Cooper pairs as the coupling strength increases [26]. Our result here suggests that the quark matter possibly realized in the core of neutron stars may be rather like the BEC of tightly bound Cooper pairs.

For better understanding of the internal structure of the quark Cooper pair, let us consider the correlation function in the coordinate space. Fig. 10 shows the spatial correlation of a Cooper pair at various chemical potentials normalized as $\int d^{3} r\left|\varphi_{+}(r)\right|^{2}=1$. As is expected, the density dependence of the quark correlation in the coordinate space is opposite to that in the momentum space. At high density, most of the quarks participating in forming a Cooper pair have the Fermi momentum $k_{\mathrm{F}}=\mu$ giving a sharp peak in the momentum space correlation. In the coordinate space, this corresponds to an oscillatory distribution with a wavelength $\lambda=1 / \mu$ without much structure near the origin. (The oscillation is also evident from the factor $\sin (\mu r)$ in the approximate correlation function Eq. (2.20) discussed in Sect. IIE.) At lower densities, accumulation of the correlation near the origin in the coordinate space is much more prominent in Fig. 10. This implies a localized Cooper pair composed of quarks with various momentum. This is also seen by the broad momentum correlation in Fig. 8(a).

(a)

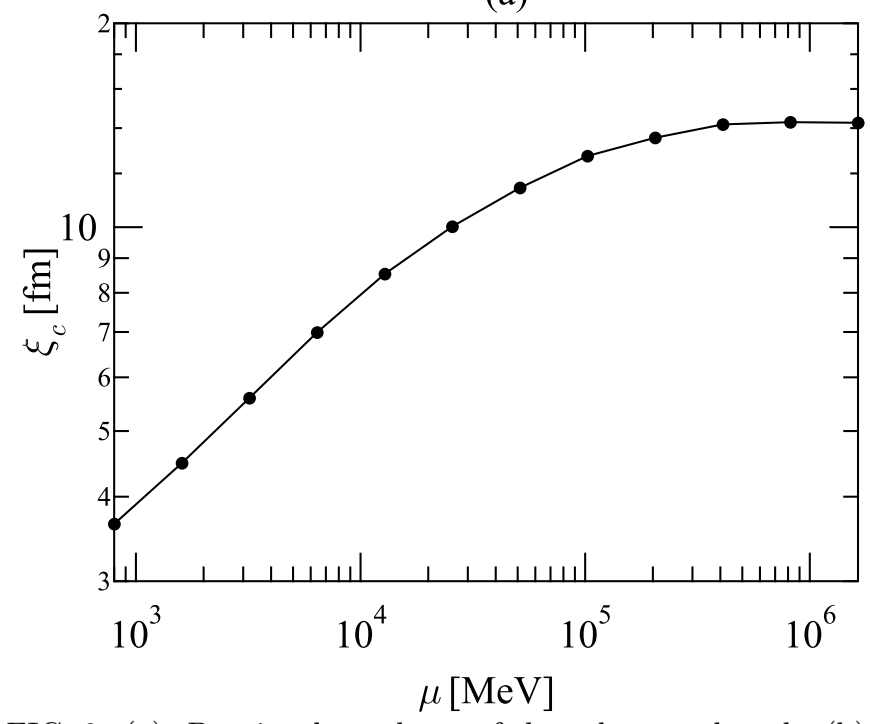

(b)

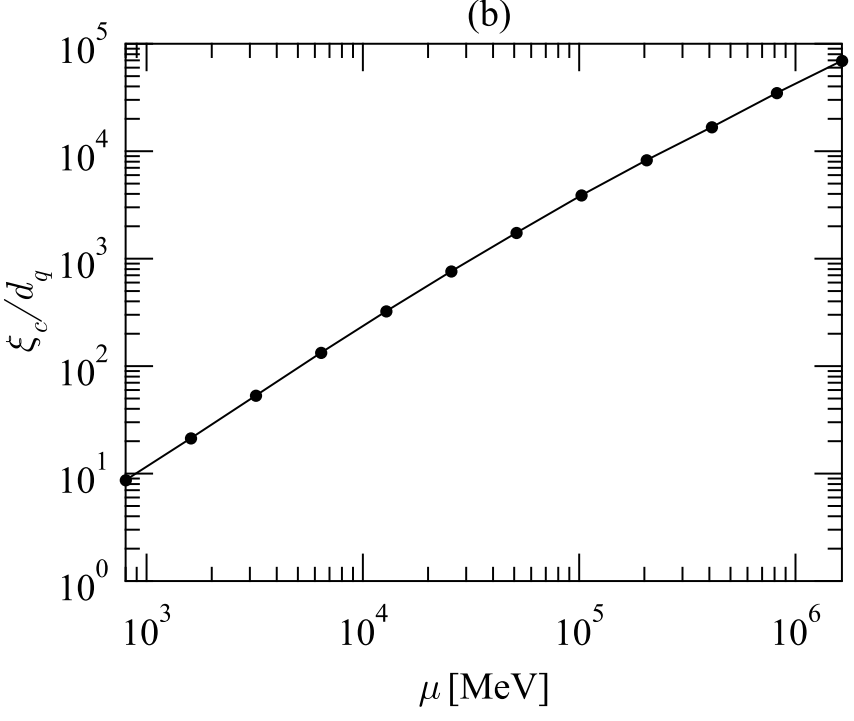

FIG. 9. (a): Density dependence of the coherence length. (b): Ratio of the coherence length $\xi_{\mathrm{c}}$ and the average inter quark distance $d_{q}$ as a function of the chemical potential.

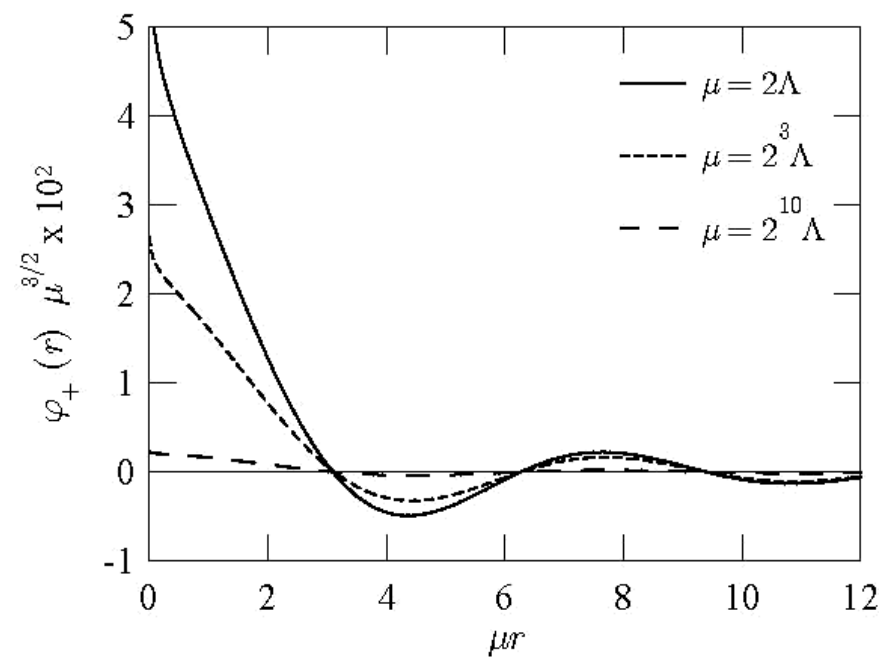

FIG. 10. The quark-quark correlation function $\varphi_{+}(r)$ in the coordinate space $\varphi_{+}(r)$ for several different chemical potentials. $\varphi_{+}(r)$ is normalized to be unity. 


\section{SUMMARY AND DISCUSSION}

In this paper, we have studied the spatial-momentum dependence of a superconducting gap and the structure of the Cooper pairs in two-flavor color superconductivity, using a single model for a very wide region of density. Nontrivial momentum dependence of the gap manifests itself at low densities, where relatively large QCD coupling allows the Cooper pairing to take place in a wide region around the Fermi surface. Our results imply that the quark matter which might exist in the core of neutron stars or in the quark stars could be rather different from that expected from the weak-coupling BCS picture.

Following is the summary of what we have discussed in this paper.

(1) At high density, the weak-coupling gap equation with the electric and magnetic gluons is a good approximation. The momentum dependent quark-gluon vertex and the coupling to the antiquark pairing do not change the weakcoupling result. The gap $\Delta_{+}(k)$ has a peak around the Fermi surface with a width $\sim 2 \Delta_{+}(\mu)$ and decreases rapidly as $k$ goes away from the Fermi surface. This is consistent with the analytic solution of the gap equation in the weak-coupling limit. All the results indicate that the Cooper pairing takes place only in a small region around the Fermi surface.

(2) At lower density, the weak-coupling gap equation is no longer a good approximation. The momentum-dependent vertex and the coupling to the antiquark pairing have non-negligible effects. The sharp peak at the Fermi surface disappears. These imply that a large number of Cooper pairs away from the Fermi surface participate in the color superconductivity. This was confirmed by the quark occupation numbers and the quark-quark correlation. Therefore, color superconductivity at low density is not a phenomenon in the vicinity of the Fermi surface, but is a phenomenon with large modification of the Fermi surface.

(3) The qualitative change of color superconductivity from high density to low density can be explicitly seen by the ratio of the size of the quark Cooper pair to the averaged inter-quark distance. At high density, the ratio is very large (about $10^{5}$ at $\mu \sim 10^{6} \mathrm{MeV}$ ) which is consistent with the standard BCS-type superconductivity. At lower densities, however, the ratio becomes small (about 10 at $\mu=800 \mathrm{MeV}$ ). This situation is rather similar to the "strong coupling" superconductor which could be described by a Bose-Einstein condensate of tightly bound Cooper pairs.

There are several future problems.

Firstly, there are still several corrections to our "full" gap equation. They include the full hard-dense-loop corrections and the Meissner effect in the gluon propagator, and also the use of the plasmino dispersion in the diagonal self-energy of the quark propagator. The latter effect induces only a sub-leading change on the gap at the Fermi surface at high density [27,28, but may have non-negligible effects at lower densities. For treating both the diagonal and off-diagonal parts of the quark self-energy, the standard Eliashberg formalism must be used [29, 13. If we include all the above effects, the gap equation with frequency and momentum as independent variables should be solved [30.

Secondly, we assumed that there is no significant vacuum effects in the present paper. If the density is close to the critical density of chiral symmetry breaking, one must consider the interplay between the quark-antiquark condensate and the quark-quark condensate [11,12,31]. This also requires us to treat the diagonal and off-diagonal components of the fermion self-energy simultaneously, which corresponds to the Hartree-Fock-Bogoliubov theory in many-body problem [32].

Thirdly, we have taken the Landau gauge $\xi=0$ in this paper. As we discussed in Sect. IID and in Appendix A, the $\xi$-dependence in the gap equation remains as far as we properly treat the momentum dependence in the gap equation. Although it is a sub-leading contribution relative to the magnetic and magnetic interactions, it is desirable to develop a gauge invariant formalism especially when one treats the low density region.

Lastly, it will be very intriguing to find a new way of describing color superconductivity at low densities. The small size Cooper pairs with coherence length comparable to the inter-quark distance suggests that BEC description may be useful as in the analogous example in condensed matter physics. An analysis along this line has been discussed in Ref. [5] using the linear sigma model for the diquark field.

\section{ACKNOWLEDGMENTS}

H.A. would like to thank T. Tatsumi for his useful comments at the early stages of the work. He is also thankful to K. Suzuki for his various supports. K.I. is grateful to R. Pisarski, D. Rischke, T. Schäfer, and D. T. Son for their critical comments and suggestions. H.A. and K.I. are thankful to M. Matsuzaki for discussion. Lastly, H.A. and T.H. acknowledge V.A. Miransky for his interests in the work. 


\section{APPENDIX A: GAUGE DEPENDENCE OF $\Delta_{+}$}

In this Appendix, we discuss the gauge parameter dependence of the gap equation for $\Delta_{+}$at high density. We write the gauge dependent contribution to the gap equation for $\Delta_{+}(k)$ as $I_{\xi}(k)$. Neglecting the antiquark pole, it becomes

$$
I_{\xi}(k)=\frac{g^{2}}{24 \pi^{2}} \int_{0}^{\infty} d q \frac{q}{k} \int_{-1}^{1} d(\cos \theta) \frac{\Delta_{+}(q)}{\sqrt{E_{+}(q)^{2}+\Delta_{+}(q)^{2}}} K_{\xi}(q, k ; \cos \theta)
$$

where $\hat{\boldsymbol{q}} \cdot \hat{\boldsymbol{k}}=\cos \theta$ and $K_{\xi}$ is defined as

$$
K_{\xi}(q, k ; x)=\xi \times \frac{2 q k}{q^{2}+k^{2}-2 q k \cos \theta}\left\{\frac{(q-k \cos \theta)(k-q \cos \theta)}{q^{2}+k^{2}-2 q k \cos \theta}-\frac{1-\cos \theta}{2}\right\} .
$$

$I_{\xi}(k)$ should be added to the right-hand side of Eq. (2.13) when $\xi \neq 0$.

Now, if we set $q=k=\mu$ before the angular integration, the kernel vanishes for all $\cos \theta$, namely $K_{\xi}(\mu, \mu ; \cos \theta)=0$. This is the standard argument that the gauge parameter dependence does not appear in the gap equation at extremely high density. However, if we first integrate over $\cos \theta$, and then take the limit $q, k \rightarrow \mu$, the result is nonzero. This can be seen explicitly by carrying out the angular integral of $K_{\xi}$ and by writing the result in terms of a variable $Y=(k / q+q / k) / 2 \geq 1:$

$$
\int_{-1}^{1} d(\cos \theta) K_{\xi}(q, k ; \cos \theta)=\xi\left(\frac{Y-1}{2} \ln \left|\frac{Y+1}{Y-1}\right|-1\right) .
$$

If we take the limit $Y \rightarrow 1$ in the right-hand side, the first term disappears, but the second term survives and gives a gauge-dependent contribution $-\xi$. One can trace back the origin of this situation by introducing a small regulator $\epsilon$ to the collinear region of the integral $\cos \theta \sim 1$. Then, the integral above becomes

$$
F(\epsilon, Y) \equiv \int_{-1}^{1-\epsilon} d(\cos \theta) K_{\xi}(q, k ; \cos \theta)=\xi\left(\frac{Y-1}{2} \ln \left|\frac{Y+1}{Y-1+\epsilon}\right|-\frac{Y-1}{Y-1+\epsilon} \frac{Y+1}{2}\right) .
$$

The first term disappears irrespective of the order of $Y \rightarrow 1$ and $\epsilon \rightarrow+0$. However, the second term vanishes only when one takes $Y \rightarrow 1$ before $\epsilon \rightarrow 0$. Namely,

$$
\begin{aligned}
& \lim _{\epsilon \rightarrow 0} \lim _{Y \rightarrow 1} F(\epsilon, Y)=0, \\
& \lim _{Y \rightarrow 1} \lim _{\epsilon \rightarrow 0} F(\epsilon, Y)=\xi .
\end{aligned}
$$

This non-commutability of the two limits arises from the fact that $F(\epsilon, Y)$ does not approach to $F(0, Y)$ uniformly in the region $Y \geq 1$.

Therefore, if we integrate over the angular variable $\cos \theta$ exactly, the gap equation for $\Delta_{+}(k)$ at high density has an extra gauge-dependent contribution from the quark-pole;

$$
I_{\xi}(k)=-\xi \frac{g^{2}}{24 \pi^{2}} \int_{0}^{\infty} d q \frac{\Delta_{+}(q)}{\sqrt{E_{+}(q)^{2}+\Delta_{+}(q)^{2}}} \frac{q}{k}\left(1-\frac{(q-k)^{2}}{4 q k} \ln \left|\frac{(k+q)^{2}}{(k-q)^{2}}\right|\right) .
$$

Compared with the leading magnetic contribution with the kernel of logarithmic singularity, the above contribution is considered to be a sub-leading effect.

\section{APPENDIX B: PIPPARD LENGTH IN THE WEAK-COUPLING LIMIT}

In this Appendix, we derive the Pippard length from the correlation function in the weak-coupling region. We use the two-point correlation function of quarks defined in Eq. (2.18)

$$
\hat{\varphi}_{+}(q)=\frac{\Delta_{+}(q)}{\sqrt{E_{+}(q)^{2}+\Delta_{+}(q)^{2}}},
$$

Since $\hat{\varphi}_{+}(q)$ is a function of $q(>0)$ only, its Fourier transformation is given by 


$$
\begin{aligned}
\varphi_{+}(r) & =\frac{N}{2 \pi^{2}} \int_{0}^{\infty} d q q^{2} j_{0}(q r) \hat{\varphi}_{+}(q) \\
& =\frac{N}{2 \pi^{2} r} \int_{0}^{\infty} d q \sin (q r) \frac{q \Delta_{+}(q)}{\sqrt{(q-\mu)^{2}+\Delta_{+}(q)^{2}}}
\end{aligned}
$$

where $j_{0}(x)=x^{-1} \sin x$ is the 0 -th order spherical Bessel function.

In the weak-coupling limit, the integral is dominated at $q \sim \mu$. Then, by replacing $\Delta_{+}(q)$ by $\Delta_{+}(\mu)$ and performing the $q$ integration approximately, one finds

$$
\begin{aligned}
\varphi_{+}(r) & \sim \frac{N}{2 \pi^{2} r} \int_{0}^{\infty} d q \sin (q r) \frac{\mu \Delta_{+}(\mu)}{\sqrt{(q-\mu)^{2}+\Delta_{+}(\mu)^{2}}} \\
& \sim \frac{N \mu^{3}}{\pi^{2}} \frac{\sin (\mu r)}{\mu r} \frac{\Delta_{+}(\mu)}{\mu} K_{0}\left(\Delta_{+}(\mu) r\right),
\end{aligned}
$$

where $K_{0}$ is the 0 -th modified Bessel function of the second kind. Using its asymptotic form

$$
K_{0}(z) \stackrel{z \rightarrow \infty}{\sim} \sqrt{\frac{\pi}{2 z}} e^{-z} \sum_{n=0}^{\infty} \frac{\Gamma(n+1 / 2)}{\Gamma(-n+1 / 2)} \frac{1}{n !(2 z)^{n}}
$$

we obtain

$$
\varphi_{+}(r) \stackrel{r \rightarrow \infty}{\sim} N \sqrt{\frac{\mu^{5} \Delta_{+}(\mu)}{2 \pi^{3}}} \frac{\sin \mu r}{(\mu r)^{3 / 2}} e^{-r /\left(\pi \xi_{\mathrm{p}}\right)},
$$

where $\xi_{\mathrm{p}} \equiv\left(\pi \Delta_{+}(\mu)\right)^{-1}$ is the Pippard length.

[1] J. C. Collins and M. J. Perry, Phys. Rev. Lett. 34, 1353 (1975).

[2] D. Bailin and A. Love, Phys. Rept. 107, 325 (1984).

[3] M. Iwasaki and T. Iwado, Phys. Lett. B 350, 163 (1995); Prog. Theor. Phys. 94, 1073 (1995).

[4] M. Alford, K. Rajagopal, and F. Wilczek, Phys. Lett. B422, 247 (1998).

[5] R. Rapp, T. Schäfer, E. V. Shuryak, and M. Velkovsky, Phys. Rev. Lett. 81, 53 (1998).

[6] For a recent review, see K. Rajagopal and F. Wilczek, "The Condensed Matter Physics of QCD", in "At the frontier of particle physics - handbook of QCD", Volume 3, Chapter 35, edited by M. Shifman (World Scientific, 2001) hep-ph/0011333.

[7] T. Schäfer and F. Wilczek, Phys. Rev. D60, 114033 (1999).

[8] D. K. Hong, V. A. Miransky, I. A. Shovkovy and L.C.R. Wijewardhana, Phys. Rev. D61, 056001 (2000), Erratum-ibid. D62 (2000) 059903.

[9] R. D. Pisarski and D. H. Rischke, Phys. Rev. D61, 074017 (2000).

[10] D. T. Son, Phys. Rev. D 59, 094019 (1999).

[11] J. Berges and K. Rajagopal, Nucl. Phys. B538, 215 (1999).

[12] G. W. Carter and D. Diakonov, Phys. Rev. D60 (1999) 016004.

[13] J. R. Schrieffer, "Theory of Superconductivity", (Benjamin, New York, 1964)

[14] R. Horie, "On Color Superconductivity in High Density Quark Matter", Master Thesis (Kyoto University, Feb. 1999),

[15] M. Matsuzaki, Phys. Rev. D62, 017501 (2000).

[16] M. Alford, K. Rajagopal, and F. Wilczek, Nucl. Phys. B537, 443 (1999).

[17] K. Higashijima, Phys. Rev. D29, 1228 (1984); Prog. Theor. Phys. Suppl. 104, 1 (1991). V. A. Miransky, Sov. J. Nucl. Phys. 38, 280 (1983).

[18] K-I. Aoki, T. Kugo and M. Mitchard, Phys. Lett. B266, 467 (1991). K-I. Aoki, M. Bando, T. Kugo and M. Mitchard, Prog. Theor. Phys. 84, 683 (1990).

[19] T. Kugo, "Basic Concepts in Dynamical Symmetry Breaking and Bound State Problems", lectures in 1991 Nagoya Spring School on Dynamical Symmetry Breaking (April 23-27, 1991, Nagoya, Japan) Ed. K. Yamawaki (World Scientific). V. A. Miransky, "Dynamical Symmetry Breaking in Quantum Field Theories" (World Scientific, 1993).

[20] M. LeBellac, "Thermal Field Theory" (Cambridge University Press, 1996).

[21] C. D. Roberts and S. M. Schmidt, Prog.Part.Nucl.Phys. 45S1, 1(2000).

[22] K. Iida and G. Baym, Phys. Rev. D63, 074018 (2001). 
[23] R. D. Pisarski and D. H. Rischke, Phys. Rev. D60, 094013 (1999).

[24] K. Rajagopal and E. Shuster, Phys. Rev. D62, 085007 (2000).

[25] A. L. Fetter and J. D. Walecka, "Quantum Theory of Many-Particle Systems" (McGraw-Hill, 1971).

[26] P. Noziéres and S. Schmitt-Rink, J. Low Temp. Phys. 59, 195 (1985). For recent references, see e.g., E. Babaev, Int. J. Mod. Phys. A16, 1175 (2001) and references thein.

[27] W. E. Brown, J. T. Liu, and H. C. Ren, Phys. Rev. D61, 114012 (2000); ibid. D62, 054013 (2000); ibid. D62, 054016 (2000).

[28] C. Manuel, Phys. Rev. D62, 114008 (2000).

[29] See e.g., D. J. Scalapino, in Superconductivity, edited by R. D. Parks (Dekker, New York, 1969).

[30] H. Abuki, T. Hatsuda, and K. Itakura, work in progress.

[31] H. Abuki, "Color Superconductivity in Quark Matter at High Density" Master Thesis (Kyoto University, Feb. 2000)

[32] P. Ring and P. Schuck, "Nuclear Many-Body Problem" (Springer, New York, 1980). 


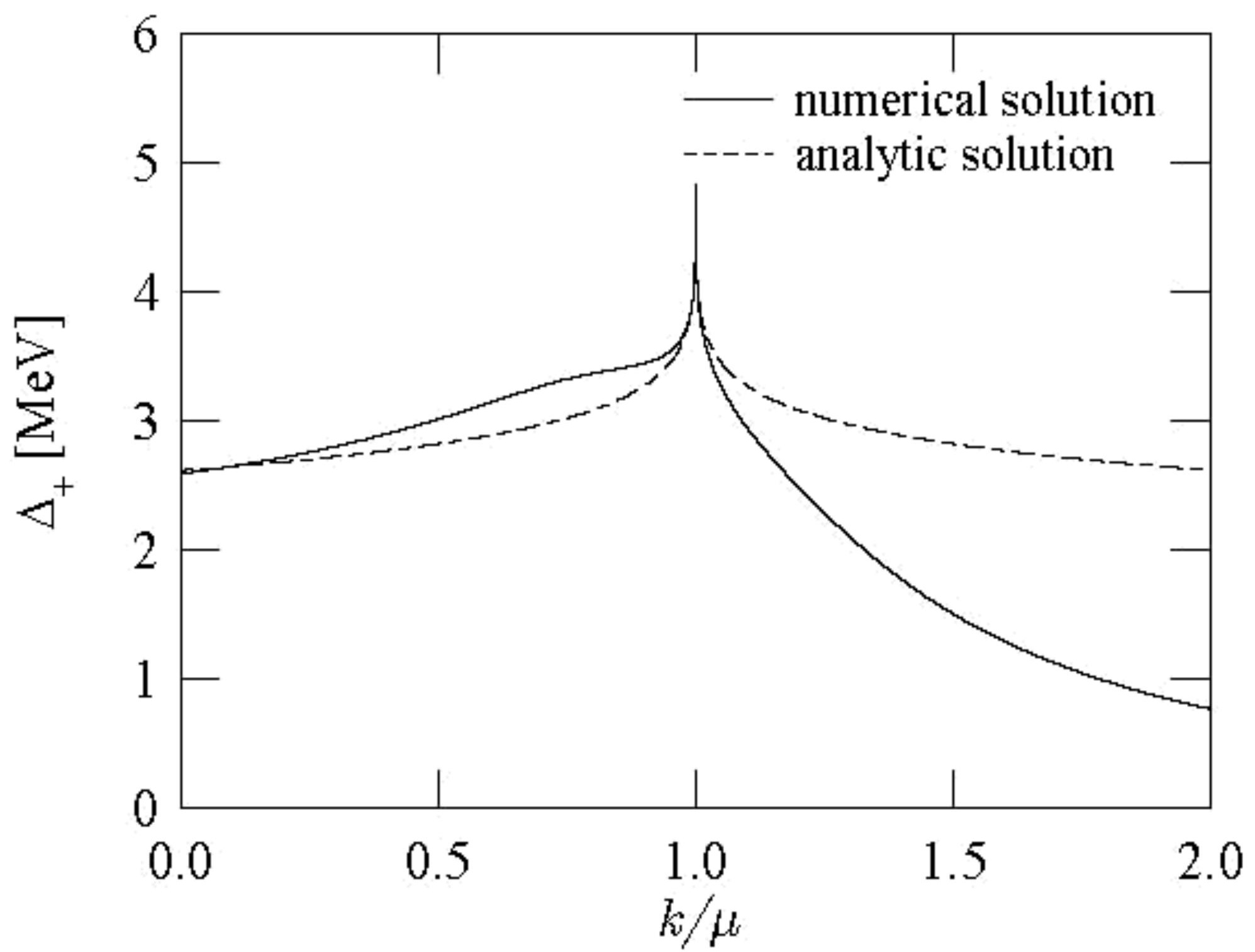

